\title{
Gestational diabetes mellitus
}

\author{
Thomas A. Buchanan' ${ }^{1}$ and Anny H. Xiang ${ }^{2}$ \\ ${ }^{1}$ Departments of Medicine, Obstetrics and Gynecology, and Physiology and Biophysics, and 2 Department of Preventive Medicine, \\ University of Southern California Keck School of Medicine, Los Angeles, California, USA.
}

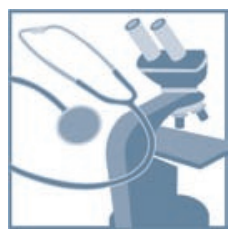

Gestational diabetes mellitus (GDM) is defined as glucose intolerance of various degrees that is first detected during pregnancy. GDM is detected through the screening of pregnant women for clinical risk factors and, among at-risk women, testing for abnormal glucose tolerance that is usually, but not invariably, mild and asymptomatic. GDM appears to result from the same broad spectrum of physiological and genetic abnormalities that characterize diabetes outside of pregnancy. Indeed, women with GDM are at high risk for having or developing diabetes when they are not pregnant. Thus, GDM provides a unique opportunity to study the early pathogenesis of diabetes and to develop interventions to prevent the disease.

\section{Historical perspective}

For more than a century, it has been known that diabetes antedating pregnancy can have severe adverse effects on fetal and neonatal outcomes (1). As early as in the 1940s, it was recognized that women who developed diabetes years after pregnancy had experienced abnormally high fetal and neonatal mortality (2). By the 1950 s the term "gestational diabetes" was applied to what was thought to be a transient condition that affected fetal outcomes adversely, then abated after delivery (3). In the 1960s, O'Sullivan found that the degree of glucose intolerance during pregnancy was related to the risk of developing diabetes after pregnancy. $\mathrm{He}$ proposed criteria for the interpretation of oral glucose tolerance tests (OGTTs) during pregnancy that were fundamentally statistical, establishing cut-off values - approximately 2 standard deviations - for diagnosing glucose intolerance during pregnancy (4). In the 1980s those cut-off points were adapted to modern methods for measuring glucose and applied to the modern definition of gestational diabetes - glucose intolerance with onset or first recognition during pregnancy (5). While based on O'Sullivan's values for predicting diabetes after pregnancy, the diagnosis of gestational diabetes mellitus (GDM) also identifies pregnancies at increased risk for perinatal morbidity (6-8) and long-term obesity and glucose intolerance in offspring (9-11).

\section{Population perspective}

Clinical detection of GDM is carried out to identify pregnancies at increased risk for perinatal morbidity and mortality. Available data do not identify a threshold of maternal glycemia at which such risk begins or increases rapidly. A multinational study, the Hyperglycemia and Adverse Pregnancy Outcome study, is underway to explore this issue in a large multiethnic cohort. In the absence of a defined glucose threshold for perinatal risk, many different sets of glycemic criteria have been proposed and are employed worldwide for the diagnosis of GDM. The criteria

Nonstandard abbreviations used: DPP, Diabetes Prevention Program; GAD, glutamic acid decarboxylase; GDM, gestational diabetes mellitus; MODY, maturityonset diabetes of the young; OGTT, oral glucose tolerance test; TRIPOD; Troglitazone in Prevention of Diabetes.

Conflict of interest: T.A. Buchanan and A.H. Xiang receive grant support from Takeda Pharmaceuticals North America Inc. T.A. Buchanan is also a consultant to Takeda Pharmaceuticals North America Inc. and is on the company's Actos Speakers' Bureau.

Citation for this article: J. Clin. Invest. 115:485-491 (2005).

doi:10.1172/JCI200524531. currently recommended by the American Diabetes Association (12) are based on O'Sullivan's criteria (see above). The detection of GDM, a condition that is generally asymptomatic, involves screening in 2 sequential steps (Tables 1 and 2), followed by administration of a 2- or 3-hour OGTT (Table 3) to women determined to be at risk by screening. Women with very high clinical risk characteristics may be diagnosed with probable pregestational (preexisting) diabetes based on the criteria provided in Table 4. When the diagnostic criteria for a 3-hour OGTT presented in Table 3 was applied to a group of Caucasian women in Toronto, approximately 7\% had GDM (6). The frequency of GDM may vary among ethnic groups (higher in groups with increased prevalence of hyperglycemia) (13-16) and with the use of different diagnostic criteria (higher when lower glucose thresholds are applied and vice versa) (4). Nonetheless, all approaches to GDM detection pinpoint - and thereby allow diagnosis of - women with glucose tolerance in the upper end of the population distribution during pregnancy. A small minority of those women have glucose levels that would be diagnostic of diabetes outside of pregnancy (Table 4). The great majority have lower glucose levels. Both groups impart to their offspring an increased risk of perinatal morbidity and long-term obesity and diabetes that appear to be related at least in part to fetal overnutrition in utero. They also incur for themselves a risk of diabetes after pregnancy that is the main focus of this paper.

\section{Etiology and pathogenesis}

\section{Normal pregnancy}

A detailed discussion of glucose regulation in pregnancy is beyond the scope of this paper. However, 2 points are important for the discussion that follows. First, pregnancy is normally attended by progressive insulin resistance that begins near mid-pregnancy and progresses through the third trimester to levels that approximate the insulin resistance seen in individuals with type 2 diabetes. The insulin resistance appears to result from a combination of increased maternal adiposity and the insulin-desensitizing effects of hormonal products of the placenta. The fact that insulin resistance rapidly abates following delivery suggests that the major contributors to this state of resistance are placental hormones. The second point is that pancreatic $\beta$ cells normally increase their insulin secretion to compensate for the insulin resistance of pregnancy (see Figure 1, for example). As a result, changes in circulating glucose levels over the course of pregnancy are quite small compared 


\section{Table 1}

Screening for GDM, step 1: clinical risk assessment ${ }^{A, B}$

$\begin{array}{ll}\text { Risk category } & \text { Clinical characteristics } \\ \text { High risk (presence of } & \text { Marked obesity } \\ \text { any is sufficient) } & \text { Diabetes in first-degree relative(s) } \\ & \begin{array}{l}\text { Personal history of glucose intolerance } \\ \text { Prior delivery of macrosomic infant } \\ \text { Current glycosuria }\end{array} \\ \text { Average risk } & \text { Fits neither low- nor high-risk profile } \\ \text { Low risk (all required) } & \text { Age <25 } \\ & \text { Low-risk ethnicityc } \\ & \text { No diabetes in first-degree relatives } \\ & \text { Normal prepregnancy weight and pregnancy weight gain } \\ & \text { No personal history of abnormal glucose levels } \\ & \text { No prior poor obstetrical outcomes }\end{array}$

\section{Risk category}

High risk (presence of

any is sufficient)

ABased on recommendations of the American Diabetes Association (5). BPerformed at initial antepartum visit. CEthnicities other than Hispanic, African Native American, South or East Asian, Pacific Islander, or indigenous Australian, all of which have relatively high rates of GDM. Adapted with permission from N. Engl. J. Med. (72). Copyright 1999, Massachussets Medical Society. All rights reserved.

\section{Recommended screening}

Blood glucose screening at initial antepartum visit or as soon as possible thereafter; repeat at 24-28 wk if not already diagnosed with GDM by that time

Blood glucose screening between 24 and 28 wk gestation Blood glucose screening not required with the large changes in insulin sensitivity. Robust plasticity of $\beta$ cell function in the face of progressive insulin resistance is the hallmark of normal glucose regulation during pregnancy.

\section{Gestational diabetes}

GDM is a form of hyperglycemia. In general, hyperglycemia results from an insulin supply that is inadequate to meet tissue demands for normal blood glucose regulation. Studies conducted during late pregnancy, when, as discussed below, insulin requirements are high and differ only slightly between normal and gestational diabetic women, consistently reveal reduced insulin responses to nutrients in women with GDM (17-23). Studies conducted before or after pregnancy, when women with prior GDM are usually more insulin resistant than normal women (also discussed below), often reveal insulin responses that are similar in the 2 groups or reduced only slightly in women with prior GDM $(18,22-26)$. However, when insulin levels and responses are expressed relative to each individual's degree of insulin resistance, a large defect in pancreatic $\beta$ cell function is a consistent finding in women with prior $\operatorname{GDM}(23,25,27)$.

Potential causes of inadequate $\beta$ cell function are myriad and not fully described. Outside of pregnancy, there are 3 general settings that are recognized - through classification as distinct forms of diabetes mellitus (12) - as separate categories of $\beta$ cell dysfunction: (a) autoimmune; (b) monogenic; and (c) occurring on a background of insulin resistance. There is evidence that $\beta$ cell dysfunction in GDM can occur in all 3 major settings, a fact that is not surprising given that GDM is detected by what is, in essence, population screening for elevated glucose levels among pregnant women.

\section{Autoimmune diabetes and GDM}

Type 1 diabetes results from autoimmune destruction of pancreatic $\beta$ cells. It accounts for approximately $5-10 \%$ of diabetes in the general population (12). Prevalence rates vary by ethnicity, with the highest rates in Scandinavians and the lowest rates (i.e., $0 \%$ ) in full-blooded Native Americans. Type 1 diabetes is characterized by circulating immune markers directed against pancreatic islets (anti-islet cell antibodies) or $\beta$ cell antigens (such as glutamic acid decarboxylase [GAD]). A small minority (less than $10 \%$ in most studies) of women with GDM have the same markers present in their circulation (17, 28-31). Although detailed physiological studies of these women are lacking, they most likely have inadequate insulin secretion resulting from autoimmune damage to and destruction of pancreatic $\beta$ cells. They appear to have evolving type 1 diabetes, which comes to clinical attention through routine glucose screening during pregnancy. The frequency of anti-islet cell and anti-GAD antibodies detected in GDM tends to parallel ethnic trends in the prevalence of type 1 diabetes outside of pregnancy. Patients with anti-islet cell or anti-GAD antibodies often, but not invariably, are lean, and they can rapidly develop overt diabetes after pregnancy (30).

\section{Monogenic diabetes and GDM}

Monogenic diabetes mellitus has been identified outside of pregnancy in 2 general forms. Some patients have mutations in autosomes (autosomal dominant inheritance pattern, commonly referred to as maturity-onset diabetes of the young [MODY], with genetic subtypes denoted as MODY 1, MODY 2, etc.). Others have mutations in mitochondrial DNA, often with distinct clinical syndromes such

Table 2

Screening for GDM, step 2: blood glucose screening ${ }^{A}$

$\begin{array}{lcc}\begin{array}{l}\text { Glucose cut } \\ \text { point }^{\text {B }}\end{array} & \begin{array}{c}\text { Percentage of women } \\ \text { with positive test }^{C}\end{array} & \begin{array}{c}\text { Sensitivity } \\ \text { for GDMC }\end{array} \\ \geq 140 \mathrm{mg} / \mathrm{dl}(7.8 \mathrm{mmol} / \mathrm{l}) & 14-18 \% & \sim 80 \% \\ \geq 130 \mathrm{mg} / \mathrm{dl}(7.2 \mathrm{mmol} / \mathrm{l}) & 20-25 \% & \sim 90 \%\end{array}$

A The test is a $50-\mathrm{g}$ oral glucose challenge performed in patients with high or average clinical risk characteristics. No preparation is needed; the test can be done any time of day. Women with very high clinical risk characteristics may proceed directly to measurement of fasting glucose or to diagnostic OGTT (see Tables 3 and 4). BVenous serum or plasma glucose measured by certified clinical laboratory. Cut point identifies women recommended for 2- or 3- hour OGTTs as described in Table 3. CMay vary with ethnicity and with diagnostic OGTT employed. Adapted with permission from N. Engl. J. Med. (72). Copyright 1999, Massachussets Medical Society. All rights reserved. 


\section{Table 3}

Diagnosis of GDM during pregnancy ${ }^{A}$

\begin{tabular}{lccc} 
Procedure & \multicolumn{3}{c}{ Glucose cut points $^{\mathrm{B}}$} \\
& Time (h) & $\mathbf{m g} / \mathbf{d l}$ & $\mathbf{m m o l} / \mathbf{l}$ \\
100-g, 3-h OGTTC & Fasting & 95 & 5.3 \\
& 1 & 180 & 10.0 \\
& 2 & 155 & 8.6 \\
75-g, 2-h 0GTTC & 3 & 140 & 7.8 \\
& Fasting & 95 & 5.3 \\
& 1 & 180 & 10.0 \\
& 2 & 155 & 8.6 \\
\hline
\end{tabular}

ABased on recommendations of the American Diabetes Association (5). BVenous serum or plasma glucose measured by a certified clinical laboratory. ${ }^{\mathrm{C}}$ Two or more values meeting or exceeding the cut points are required for diagnosis. Adapted with permission from N. Engl. J. Med. (72). Copyright 1999, Massachussets Medical Society. All rights reserved.

as deafness. In both instances, onset tends to occur at an early age relative to other forms of nonimmune diabetes (e.g., type 2 diabetes, described below), and patients tend not to be obese or insulin resistant. Both features point to abnormalities in the regulation of $\beta$ cell mass and/or function. Indeed, detailed metabolic studies have revealed abnormalities in glucose-mediated insulin secretion in some forms of MODY (32). Mutations that cause several subtypes of MODY have been found in women with GDM. These include mutations in genes coding for: (a) glucokinase (MODY 2) (29, 33-35); (b) hepatocyte nuclear factor $1 \alpha$ (MODY 3) (29); (c) and insulin promoter factor 1 (MODY 4) (29). Together, these monogenic forms of GDM account for less than $10 \%$ of GDM cases $(29,33-36)$. They likely represent cases of preexisting diabetes that are first detected by routine glucose screening during pregnancy.

\section{Insulin resistance, $\beta$ cell dysfunction, and GDM}

The majority of women with GDM appear to have $\beta$ cell dysfunction that occurs on a background of chronic insulin resistance. As noted above, pregnancy normally induces quite marked insulin resistance. This physiological insulin resistance also occurs in women with GDM. However, it occurs on a background of chronic insulin resistance to which the insulin resistance of pregnancy is partially additive. As a result, pregnant women with GDM tend to have even greater insulin resistance than normal pregnant women. Differences in whole-body insulin sensitivity tend to be small in the third trimester, owing to the marked effects of pregnancy itself on insulin resistance. Nonetheless, precise and direct measures of insulin sensitivity applied during the third trimester have identified, in women with GDM, exaggerated resistance to insulin's ability to stimulate glucose utilization $(17,18)$ and to suppress both glucose production $(17,18)$ and fatty acid levels $(17)$. After delivery, when the acquired insulin resistance of pregnancy abates, women who had GDM end up, on average, with considerably greater insulin resistance than normal women. This finding, which has been consistent across studies in which whole-body insulin sensitivity has been measured directly $(22,23,25,26,37-40)$, indicates that most women who develop GDM have chronic insulin resistance. Sequential measurements of insulin sensitivity performed in the same women before pregnancy, early in the second trimester, and in the third trimester have documented insulin resistance in both lean and obese women who go on to develop GDM $(18,24)$.

Only a small number of potential biochemical mediators of the chronic insulin resistance that frequently accompanies GDM and that likely contributes to the high risk of type 2 diabetes have been examined. It is likely that there is not a single underlying biochemical etiology. Women with GDM tend to be obese, so mechanisms promoting obesity and/or linking obesity to insulin resistance are likely to play a role. Small studies have revealed increased circulating levels of leptin (41) and the inflammatory markers TNF- $\alpha$ (42) and C-reactive protein $(43)$ and decreased levels of adiponectin $(44,45)$ in women with GDM. Increased content of fat in liver (46) and muscle (47) has also been reported in women with previous gestational diabetes. All of these findings are consistent with the current understanding of some potential causes of obesity-related insulin resistance.

Defects in the binding of insulin to its receptor in skeletal muscle do not appear to be involved in the exaggerated insulin resistance of GDM (48). Alterations in the insulin signaling pathway (49-52), abnormal subcellular localization of GLUT4 transporters (53), reduced expression of PPAR $\gamma(49)$, increased expression of the membrane glycoprotein PC-1 (51), and reduced insulin-mediated glucose transport $(52,53)$ have been found in skeletal muscle or fat cells of women with GDM or a history thereof compared with normal women. Whether any of these defects is primary or the result of more fundamental defects in insulin action is currently unknown. Given that GDM represents a cross-section of young women with glucose intolerance, mechanisms that lead to chronic insulin resistance in GDM are likely to be as varied as they are in the general population.

It has long been thought (and taught) that GDM develops in women who cannot increase their insulin secretion when faced with the increased insulin needs imposed by late pregnancy. Serial studies of women who develop GDM do not support that concept. As illustrated in Figure 1, insulin secretion in obese women who develop GDM can increase considerably over weeks or months in association with the acquired insulin resistance of pregnancy. However, the increase occurs along an insulin sensitivity-secretion curve that is approximately 50\% lower (i.e., $50 \%$ less insulin for any degree of insulin resistance) than that of normal women. These short-term responses appear to occur on a background of long-term deterioration of $\beta$ cell function that, over years, leads to progressive hyperglycemia and diabetes (see "Link to diabetes after pregnancy," below). Longitudinal studies of lean and obese women before pregnancy, at the beginning of the second trimester, and in the third trimester also reveal an increase in insulin secretion in association with the acquired insulin resistance of pregnancy $(18,24)$. However, the increase is less than that which occurs in normal pregnant women despite somewhat greater insulin resis-

Table 4

Probable pregestational diabetes ${ }^{A}$

$\begin{array}{ll}\text { Timing of sample } & \text { Serum or plasma glucose } \\ \text { After overnight fast } & \geq 126 \mathrm{mg} / \mathrm{dl}(7.0 \mathrm{mmol} / \mathrm{l}) \\ \text { Random } & \geq 200 \mathrm{mg} / \mathrm{dl}(11.1 \mathrm{mmol} / \mathrm{l})\end{array}$

${ }^{A}$ Based on recommendations of the American Diabetes Association (5). BVenous serum or plasma glucose measured by a certified clinical laboratory. Adapted with permission from N. Engl. J. Med. (72). Copyright 1999, Massachussets Medical Society. All rights reserved. 


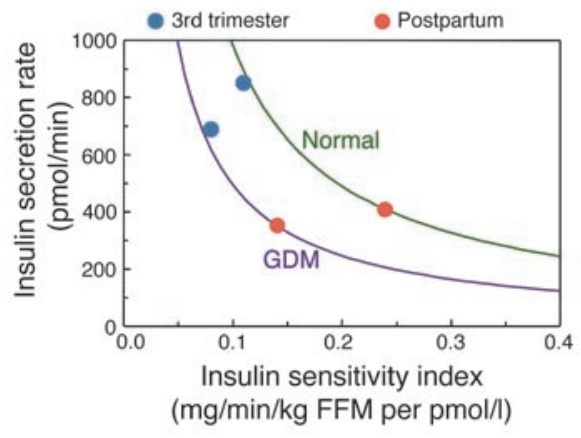

Figure 1

Insulin sensitivity-secretion relationships in women with GDM and normal women during the third trimester and remote from pregnancy. Values were measured at the end of 3-hour hyperglycemic clamps (plasma glucose, about $180 \mathrm{mg} / \mathrm{dl}$ ) (22). Prehepatic insulin secretion rates were calculated from steady-state plasma insulin and C-peptide levels. Insulin sensitivity index was calculated as steady-state glucose infusion rate divided by steady-state plasma insulin concentration. FFM, fat-free mass. Figure reproduced with permission from J. Clin. Endocrinol. Metab. (27). Copyright 2001, The Endocrine Society.

tance in individuals with GDM. These small but elegant physiological studies reveal that the limitation in insulin secretion in women with GDM is not necessarily fixed. Rather, in at least some of them, insulin secretion is low relative to their insulin sensitivity but responsive to changing sensitivity. One approach to the prevention of diabetes after GDM has taken advantage of this responsiveness (discussed below in "Link to diabetes after pregnancy").

Very little is known about the genetics of GDM in women with chronic insulin resistance. The few studies that have been done have compared allele frequencies of candidate genes in women with and without GDM, with no selection for specific phenotypic subtypes of GDM. Variants that differed in frequency between control and GDM subjects were found in genes coding for: (a) the islet-specific promoter of glucokinase (54), known to be important for glucose sensing by $\beta$ cells; (b) calpain-10 (55), a gene associated with type 2 diabetes in Hispanic Americans and some other ethnic groups; (c) the sulfonylurea receptor 1 (56), which is involved in glucose-stimulated insulin secretion; and (d) the $\beta 3$ adrenoreceptor, which may regulate body composition. Whether these findings will be confirmed in larger studies with broader representation among women with GDM remains to be determined.

\section{Link to diabetes after pregnancy}

The hyperglycemia of GDM is detected at one point in a women's life. If glucose levels are not already in the diabetic range, GDM could represent glucose intolerance that is limited to pregnancy, is chronic but stable, or is at a stage in the progression to diabetes. Long-term follow-up studies, recently reviewed by Kim et al. (57), reveal that most, but not all, women with GDM do progress to diabetes after pregnancy. Only approximately $10 \%$ of patients have diabetes soon after delivery (58). Incident cases appear to occur at a relatively constant rate during the first 10 years thereafter (57), and the few studies that have been conducted over a period of more than 10 years reveal a stable long-term risk of approximately $70 \%$ (57).

Most studies of risk factors for the development of diabetes after GDM fail to distinguish among the possible subtypes of GDM and diabetes discussed above. They generally reveal risk factors, such as obesity, weight gain, and increased age, that are known to be associated with type 2 diabetes. Relatively high glucose levels during and soon after pregnancy also correlate with increased risk of diabetes, perhaps because they identify women who are relatively close to developing diabetes when the diagnosis of GDM is made.

Longitudinal studies of the pathophysiology of diabetes that develops after GDM are limited to Hispanic women with clinical characteristics suggesting a risk for type 2 diabetes. Those studies have revealed much about the $\beta$ cell defect that leads to type 2 diabetes after GDM in 1 ethnic group. First, weight gain and additional pregnancies, factors associated with chronic and acute insulin resistance, respectively, independently increase the risk of developing diabetes (59). Second, decreasing $\beta$ cell function is associated with increasing hyperglycemia (Figure 2) (60). The impact of reduced $\beta$ cell function on glucose levels is relatively small until the disposition index, which reflects acute insulin responses to glucose in relation to insulin resistance, is very low (approximately $10-15 \%$ of normal). Thereafter, relatively small differences in $\beta$ cell function are associated with relatively large increases in glucose levels (60). Third, treatment of insulin resistance at the stage of impaired glucose tolerance results in a reciprocal downregulation of insulin secretion (61), which in turn is associated with a reduction in the risk of diabetes and with preservation of $\beta$ cell function (62). Taken together, these 3 findings reveal a progressive loss of insulin secretion that appears to be caused by high insulin secretory demands imposed by chronic insulin resistance. Glycemia in the diabetic range is a relatively late consequence of the loss of insulin secretion. That loss can be slowed or stopped through the treatment of insulin resistance in order to reduce of high insulin secretory demands (62). Whether the same or similar mechanisms of progressive $\beta$ cell dysfunction and opportunities for $\beta$ cell preservation occur in other ethnic groups remains to be determined.

\section{Implications for clinical care}

To date, insights into the mechanisms underlying impaired glucose regulation in GDM have not had an important impact on clinical management during pregnancy. The focus for antepartum care is on the use of standard antidiabetic treatments, mostly appropriate nutrition and exogenous insulin delivery but more recently administration of selected oral antidiabetic agents (63-65), to normalize maternal pre- and postprandial glucose levels and minimize fetal overnutrition. Fetal ultrasound measurements have also been used to refine the identification of pregnancies in which the fetus demonstrates signs of excessive adiposity - pregnancies in which the need to aggressively lower maternal glucose is the greatest $(66,67)$.

After pregnancy, the main focus of clinical care should be on reducing the risk of diabetes and detecting and treating diabetes that does develop. Measurement of fasting glucose in the immediate postpartum period will identify women with persistent fasting hyperglycemia in the diabetic range. Other women should have an OGTT sometime during the first 2-6 months postpartum and, if not diabetic, annual testing for diabetes. Family planning is important to reduce the occurrence of unplanned pregnancies in the presence of poorly controlled diabetes (68), a scenario that leads to serious birth defects in offspring (69).

Classification of patients into 1 of the 3 major subtypes of GDM discussed in this review can aid in clinical management. Lean patients are less likely to be insulin resistant than overweight or obese patients, so autoimmune and monogenic forms of diabetes should be considered in such patients. Screening for evolving autoimmune diabetes 


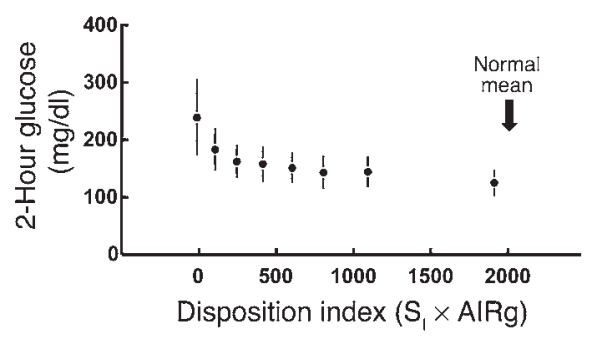

Figure 2

Relationship between pancreatic $\beta$ cell function and post-challenge glucose levels in women with prior GDM. Data are from 71 nonpregnant Hispanic women who had at least 2 ( $86 \%$ had at least 3$)$ sets of oral and frequently sampled i.v. glucose tolerance tests that were scheduled at 15-month intervals between 15 and 75 months after the index pregnancy (totaling 280 sets of tests). Participants had fasting plasma glucose of less than $140 \mathrm{mg} / \mathrm{dl}$ at entry into the study and were followed until that value was exceeded. Disposition index ( $x$ axis) is the product of minimal model insulin sensitivity $\left(\mathrm{S}_{\mathrm{I}}\right)$ and the acute insulin response to i.v. glucose (AIRg), a measure of pancreatic $\beta$ cell compensation for insulin resistance. The $y$ axis shows glucose values at hour 2 of $75-\mathrm{g}$ OGTTs. Symbols represent mean values for disposition index and corresponding 2-hour glucose values ( $\pm 1 \mathrm{SD}$ ) in each octile of disposition index. The mean disposition index was 2018 in Hispanic women without a history of GDM (arrow). Figure based on data from ref. 60.

by measuring antibodies to GAD may be warranted, particularly in women with no strong family history of diabetes who are from ethnic groups in which type 1 diabetes is relatively common. Although there are no established treatments to modify the progression to type 1 diabetes, careful monitoring of glucose levels is advised because patients can rapidly develop diabetes after pregnancy (30). Genotyping for monogenic diabetes is still primarily a research tool, but clinical tests are being developed. Early-onset diabetes with a relevant family history (autosomal dominant inheritance for MODY; maternal inheritance for mitochondrial mutations) may provide a clue to the diagnosis. In addition, Ellard et al. (34) have provided 4 clinical criteria that have relatively high specificity for identifying women with the glucokinase mutations that cause 1 form of MODY, MODY2: (a) persisting fasting hyperglycemia $(105-145 \mathrm{mg} / \mathrm{dl})$ after pregnancy; (b) a small (less than $82 \mathrm{mg} / \mathrm{dl}$ ) increment in glucose above the fasting level during a 75-g, 2-hour OGTT; (c) insulin treatment during at least 1 pregnancy but subsequently controlled on diet; and (d) a firstdegree relative with type 2 diabetes, GDM, or fasting serum or plasma glucose greater than $100 \mathrm{mg} / \mathrm{dl}$. The constellation was infrequent in patients in the United Kingdom, but $80 \%$ of women who met all 4 criteria had glucokinase mutations. Identification of monogenic forms of diabetes is important for genetic counseling.

There is currently no clinical role for genetic testing for variants that have been associated with polygenic forms of type 2 diabetes (see "Insulin resistance, $\beta$ cell dysfunction, and GDM," above). The variants cannot be used reliably to discriminate between normal individuals and individuals affected with diabetes and, just as importantly, testing is not available to clinicians. On the other hand, recent advances in the understanding of mechanistic links between GDM and type 2 diabetes have been translated into clinical care aimed at reducing the risk of diabetes. At least 2 studies of diabetes prevention in high-risk individuals have included women with a history of GDM. In the US Diabetes Prevention Program (DPP) (70), intensive lifestyle modification to promote weight loss and increase physical activity resulted in a $58 \%$ reduction in the risk of type 2 diabetes in adults with impaired glucose tolerance. GDM was one of the risk factors that led to inclusion in the study. Protection against diabetes was observed in all ethnic groups. Treatment with metformin in the same study also reduced the risk of diabetes, but to a lesser degree and primarily in the youngest and most overweight participants. To date, specific results from women with a history of GDM have not been published.

The Troglitazone in Prevention of Diabetes (TRIPOD) study was conducted exclusively on Hispanic women with recent GDM. Assignment to treatment with the insulin-sensitizing drug troglitazone was associated with a 55\% reduction in the incidence of diabetes. Protection from diabetes was closely linked to initial reductions in endogenous insulin requirements and ultimately associated with stabilization of pancreatic $\beta$ cell function (62). Stabilization of $\beta$ cell function was also observed when troglitazone treatment was started at the time of initial detection of diabetes by annual glucose tolerance testing (71). The DPP and TRIPOD studies support clinical management that focuses on aggressive treatment of insulin resistance to reduce the risk of type 2 diabetes and, at least in Hispanic women, to preserve pancreatic $\beta$ cell function.

Taken together, these 2 studies suggest that postpartum management of women with clinical characteristics suggesting a risk for type 2 diabetes should focus on treatment of insulin resistance and monitoring of glycemia both to assess success (as reflected by stabilization of glucose levels) and to detect diabetes if it develops.

\section{Future directions}

Considerable work is needed to dissect the various mechanisms underlying maternal GDM and its evolution to diabetes after pregnancy. Large studies of screening for evolving autoimmune diabetes are necessary to more accurately define the clinical characteristics of women who need such screening as part of routine GDM management. Genetic studies may help identify women whose $\beta$ cells will tolerate insulin resistance poorly, as well as women who develop poor insulin secretion for reasons unrelated to insulin resistance. Studies of gene-environment interactions and additional studies of insulin action in muscle and fat may identify causes of insulin resistance, especially as they relate to obesity. Better understanding of mechanisms that can lead to GDM should allow more rational development and administration of therapy during pregnancy, as well as more rational approaches to prevention of both GDM and diabetes after pregnancy. GDM is an especially attractive target for such studies because the disease is detected in the course of routine clinical care and it provides an opportunity to study relatively early stages of glucose dysregulation that may be fundamental to the long-term pathobiology of diabetes.

\section{Acknowledgments}

We thank our long-term collaborators Siri Kjos, Ruth Peters, and Richard Bergman for their contributions to studies on the pathogenesis of type 2 diabetes after GDM in Hispanic women. Our work cited in this paper was supported by research grants from the NIH (R01-DK46374, R01-DK61628, and M01-RR00043), the American Diabetes Association (Clinical Research Award and Distinguished Clinical Scientist Award), and Parke-Davis Pharmaceutical Research (the TRIPOD study).

Address correspondence to: Thomas A. Buchanan, Room 6602 GNH, 1200 North State Street, Los Angeles, California 900899317, USA. Phone: (323) 226-4632; Fax: (323) 226-2796; E-mail: buchanan@usc.edu. 
1. Duncan, M. 1882. On puerperal diabetes. Trans. Obstet. Soc. Lond. 24:256-285.

2. Miller, H.C. 1946. The effect of diabetic and prediabetic pregnancies on the fetus and newborn infant. J. Pediatr. 26:455-461.

3. Carrington, E.R., Shuman, C.R., and Reardon, H.S 1957. Evaluation of the prediabetic state during pregnancy. Obstet. Gynecol. 9:664-669.

4. O'Sullivan, J.B., and Mahan, C.M. 1964. Criteria for the oral glucose tolerance test in pregnancy. Diabetes. 13:278-285.

5. American Diabetes Association. 2003. Report of the expert committee on the diagnosis and classification of diabetes mellitus. Diabetes Care. 26(Suppl. 1):S5-S20.

6. Naylor, C.D., Sermer, M., Chen, E., and Sykora, K. 1996. Cesarean delivery in relation to birth weight and gestational glucose tolerance: pathophysiology or practice style? JAMA. 275:1165-1170.

7. Magee, M.S., Walden, C.E., Benedetti, T.J., and Knopp, R.H. 1993. Influence of diagnostic criteria on the incidence of gestational diabetes and perinatal morbidity. JAMA. 269:609-615.

8. Schmidt, M.I., et al. 2001. Gestational diabetes mellitus diagnosed with a 2 -h 75 -g oral glucose tolerance test and adverse pregnancy outcomes. Diabetes Care. 24:1151-1155.

9. Pettitt, D.J., and Knowler, W.C. 1998. Long-term effects of the intrauterine environment, birth weight, and breast-feeding on Pima Indians. Diabetes Care. 21(Suppl. 2):B138-B141.

10. Silverman, B.L., Rizzo, T.A., Cho, N.H., and Metzger, B.E. 1998. Long-term effects of the intrauterine environment. The Northwestern University Diabetes in Pregnancy Center. Diabetes Care. 21(Suppl. 2):B142-B149.

11. Vohr, B.R., McGarvey, S.T., and Tucker, R. 1999. Effects of maternal gestational diabetes on offspring adiposity at 4-7 years of age. Diabetes Care. 22:1284-1291.

12. American Diabetes Association. 2004. Diagnosis and classification of diabetes mellitus. Diabetes Care. 27(Suppl. 1):S5-S10.

13. King, H. 1998. Epidemiology of glucose intolerance and gestational diabetes in women of childbearing age. Diabetes Care. 21(Suppl. 2):B9-B13.

14. Ben-Haroush, A., Yogev, Y., and Hod, M. 2004 Epidemiology of gestational diabetes mellitus and its association with Type 2 diabetes. Diabet. Med. 21:103-113.

15. Weijers, R.N., Bekedam, D.J., and Smulders, Y.M. 2002. Determinants of mild gestational hyperglycemia and gestational diabetes mellitus in a large Dutch multiethnic cohort. Diabetes Care. 25:72-77.

16. Gunton, J.E., Hitchman, R., and McElduff, A. 2001. Effects of ethnicity on glucose tolerance, insulin resistance and beta cell function in 223 women with an abnormal glucose challenge test during pregnancy. Aust. N. Z. J. Obstet. Gynecol. 41:182-186.

17. Xiang, A.H., et al. 1999. Multiple metabolic defects during late pregnancy in women at high risk for type 2 diabetes mellitus. Diabetes. 48:848-854.

18. Catalano, P.M., Huston, L., Amini, S.B., and Kalhan, S.C. 1999. Longitudinal changes in glucose metabolism during pregnancy in obese women with normal glucose tolerance and gestational diabetes. Am. J. Obstet. Gynecol. 180:903-916.

19. Catalano, P.M., Tyzbir, E.D., Roman, N.M., Amini, S.B., and Sims, E.A. 1991. Longitudinal changes in insulin release and insulin resistance in nonobese pregnant women. Am. J. Obstet. Gynecol. 165:1667-1672.

20. Yen, S.C.C., Tsai, C.C., and Vela, P. 1971. Gestational diabetogenesis: quantitative analysis of glucose-insulin interrelationship between normal pregnancy and pregnancy with gestational diabetes. Am. J. Obstet. Gynecol. 111:792-800.
21. Buchanan, T.A., Metzger, B.E., Freinkel, N., and Bergman, R.N. 1990. Insulin sensitivity and B-cell responsiveness to glucose during late pregnancy in lean and moderately obese women with normal glucose tolerance or mild gestational diabetes. Am. J. Obstet. Gynecol. 162:1008-1014.

22. Homko, C., Sivan, E., Chen, X., Reece, E.A., and Boden, G. 2001. Insulin secretion during and after pregnancy in patients with gestational diabetes mellitus. J. Clin. Endocrinol. Metab. 86:568-573.

23. Kautzky-Willer, A., et al. 1997. Pronounced insulin resistance and inadequate betacell secretion characterize lean gestational diabetes during and after pregnancy. Diabetes Care. 20:1717-1723.

24. Catalano, P.M., et al. 1993. Carbohydrate metabolism during pregnancy in control subjects and women with gestational diabetes. Am. J. Physiol. 264:E60-E67.

25. Ryan, E.A., et al. 1995. Defects in insulin secretion and action in women with a history of gestational diabetes. Diabetes. 44:506-512.

26. Osei, K., Gaillard, T.R., and Schuster, D.P. 1998. History of gestational diabetes leads to distinct metabolic alterations in nondiabetic AfricanAmerican women with a parental history of type 2 diabetes. Diabetes Care. 21:1250-1257.

27. Buchanan, T.A. 2001. Pancreatic B-cell defects in gestational diabetes: implications for the pathogenesis and prevention of type 2 diabetes. J. Clin. Endocrinol. Metab. 86:989-993.

28. Petersen, J.S., et al. 1996. GAD65 autoantibodies in women with gestational or insulin dependent diabetes mellitus diagnosed during pregnancy. Diabetologia. 39:1329-1333.

29. Weng, J., et al. 2002. Screening for MODY mutations, GAD antibodies, and type 1 diabetes-associated HLA genotypes in women with gestational diabetes mellitus. Diabetes Care. 25:68-71.

30. Mauricio, D., et al. 1992. Islet cell antibodies identify a subset of gestational diabetic women with higher risk of developing diabetes shortly after pregnancy. Diabetes Nutr. Metab. 5:237-241.

31. Catalano, P.M., Tyzbir, E.D., and Sims, E.A.H. 1990. Incidence and significance of islet cell antibodies in women with previous gestational diabetes. Diabetes Care. 13:478-482

32. Fajans, S.S., Bell, G.I., and Polonsky, K.S. 2001 Molecular mechanisms and clinical pathophysiology of maturity-onset diabetes of the young. N. Engl. J. Med. 345:971-980.

33. Kousta, E., et al. 2001. Glucokinase mutations in a phenotypically selected multiethnic group of women with a history of gestational diabetes. Diabet. Med. 18:683-684.

34. Ellard, S., et al. 2000. A high prevalence of glucokinase mutations in gestational diabetic subjects selected by clinical criteria. Diabetologia. 43:250-253.

35. Saker, P.J., et al. 1996. High prevalence of a missense mutation of the glucokinase gene in gestational diabetic patients due to a founder-effect in a local population. Diabetologia. 39:1325-1328.

36. Chen, Y., Liao, W.X., Roy, A.C., Loganath, A., and Ng, S.C. 2000. Mitochondrial gene mutations in gestational diabetes mellitus. Diabetes Res. Clin. Pract. 48:29-35.

37. Ward, W.K., et al. 1985. Insulin resistance and impaired insulin secretion in subjects with a history of gestational diabetes mellitus. Diabetes. 34:861-869.

38. Ward, W.K., Johnston, C.L.W., Beard, J.C., Benedetti, T.J., and Porte, D., Jr. 1985. Abnormalities of islet $B$ cell function, insulin action and fat distribution in women with a history of gestational diabetes: relation to obesity. J. Clin. Endocrinol. Metab. 61:1039-1045

39. Catalano, P.M., et al. 1986. Subclinical abnormalities of glucose metabolism in subjects with previ- ous gestational diabetes. Am. J. Obstet. Gynecol. 155:1255-1263.

40. Damm, P., Vestergaard, H., Kuhl, C., and Pedersen, O. 1996. Impaired insulin-stimulated nonoxidative glucose metabolism in glucose-tolerant women with previous gestational diabetes. Am. J. Obstet. Gynecol. 174:722-729.

41. Kautzky-Willer, A., et al. 2001. Increased plasma leptin in gestational diabetes. Diabetologia. 44:164-172.

42. Winkler, G., et al. 2002. Tumor necrosis factor system and insulin resistance in gestational diabetes. Diabetes Res. Clin. Pract. 56:93-99.

43. Retnakaran, R., et al. 2003. C-reactive protein and gestational diabetes: the central role of maternal obesity. J. Clin. Endocrinol. Metab. 88:3507-3512.

44. Retnakaran, R., et al. 2004. Reduced adiponectin concentration in women with gestational diabetes: a potential factor in progression to type 2 diabetes. Diabetes Care. 27:799-800.

45. Williams, M.A., et al. 2004. Plasma adiponectin concentrations in early pregnancy and subsequent risk of gestational diabetes mellitus. J. Clin. Endocrinol. Metab. 89:2306-2311.

46. Tiikkainen, M., et al. 2002. Liver-fat accumulation and insulin resistance in obese women with previous gestational diabetes. Obes. Res. 10:859-867.

47. Kautzky-Willer, A., et al. 2003. Increased intramyocellular lipid concentration identifies impaired glucose metabolism in women with previous gestational diabetes Diabetes. 52:244-251.

48. Damm, P., et al. 1993. Insulin receptor binding and tyrosine kinase activity in skeletal muscle from normal pregnant women and women with gestational diabetes. Obstet. Gynecol. 82:251-259.

49. Catalano, P.M., et al. 2002. Downregulated IRS-1 and PPARgamma in obese women with gestational diabetes: relationship to FFA during pregnancy. Am. J. Physiol. 282:E522-E533.

50. Shao, J., Yamashita, H., Qiao, L., Draznin, B., and Friedman, J.E. 2002. Phosphatidylinositol 3-kinase redistribution is associated with skeletal muscle insulin resistance in gestational diabetes mellitus. Diabetes. 51:19-29.

51. Shao, J., et al. 2000. Decreased insulin receptor tyrosine kinase activity and plasma cell membrane glycoprotein-1 over expression in skeletal muscle from obese women with gestational diabetes (GDM): evidence for increased serine/threonine phosphorylation in pregnancy and GDM. Diabetes. 49:603-610.

52. Friedman, J.E., et al. 1999. Impaired glucose transport and insulin receptor tyrosine phosphorylation in skeletal muscle from obese women with gestational diabetes. Diabetes. 48:1807-1814.

53. Garvey, W.T., et al. 1993. Multiple defects in the adipocyte glucose transport system cause cellular insulin resistance in gestational diabetes. Diabetes. 42:1773-1785.

54. Zaidi, F.K., et al. 1997. Homozygosity for a common polymorphism in the islet-specific promoter of the glucokinase gene is associated with a reduced early insulin response to oral glucose in pregnant women. Diabet. Med. 14:228-234.

55. Leipold, H., et al. 2004. Calpain-10 haplotype combination and association with gestational diabetes mellitus. Obstet. Gynecol. 103:1235-1240.

56. Rissanen, J., et al. 2000. Sulfonylurea receptor 1 gene variants are associated with gestational diabetes and type 2 diabetes but not with altered secretion of insulin. Diabetes Care. 23:70-73.

57. Kim, C., Newton, K.M., and Knopp, R.H. 2002. Gestational diabetes and the incidence of type 2 diabetes. Diabetes Care. 25:1862-1868.

58. Kjos, S.L., et al. 1990. Gestational diabetes mellitus: the prevalence of glucose intolerance and diabetes mellitus in the first two months postpartum. Am. J. Obstet. Gynecol. 163:93-98.

59. Peters, R.K., Kjos, S.L., Xiang, A., and Buchanan, 
T.A. 1996. Long-term diabetogenic effect of a single pregnancy in women with prior gestational diabetes mellitus. Lancet. 347:227-230.

60. Buchanan, T.A., et al. 2003. Changes in insulin secretion and sensitivity during the development of type 2 diabetes after gestational diabetes in Hispanic women. Diabetes. 52(Suppl. 1):A34

61. Buchanan, T.A., et al. 2000. Response of pancreatic B-cells to improved insulin sensitivity in women at high risk for type 2 diabetes. Diabetes. 49:782-788.

62. Buchanan, T.A., et al. 2002. Preservation of pancreatic B-cell function and prevention of type 2 diabetes by pharmacological treatment of insulin resistance in high-risk Hispanic women. Diabetes. 51:2769-2803.

63. Langer, O., Conway, D.L., Berkus, M.D., Xenakis, E.M., and Gonzales, O. 2000. A comparison of glyburide and insulin in women with gestational diabetes mellitus. N. Engl. J. Med. 343:1134-1138.
64. Hellmuth, E., Damm, P., and Molsted-Pedersen, L. 2000. Oral hypoglycaemic agents in 118 diabetic pregnancies. Acta. Obstet. Gynecol. Scand. 79:958-962.

65. Glueck, C.J., Goldenberg, N., Streicher, P., and Wang, P. 2003. Metformin and gestational diabetes. Curr. Diab. Rep. 3:303-312.

66. Kjos, S.L., et al. 2001. A randomized controlled trial utilizing glycemic plus fetal ultrasound parameters vs glycemic parameters to determine insulin therapy in gestational diabetes with fasting hyperglycemia. Diabetes Care. 24:1904-1910.

67. Buchanan, T.A., et al. 1994. Use of fetal ultrasound to select metabolic therapy for pregnancies complicated by mild gestational diabetes. Diabetes Care. 17:275-283.

68. Kjos, S.L., Peters, R.K., Xiang, A., Schaefer, U., and Buchanan, T.A. 1998. Hormonal choices after gestational diabetes: subsequent pregnancy, contra- ception and hormone replacement. Diabetes Care. 21(Suppl. 2):B50-B57.

69. Towner, D., et al. 1995. Congenital malformations in pregnancies complicated by non-insulin-dependent diabetes mellitus: increased risk from poor maternal metabolic control but not from exposure to sulfonylurea drugs. Diabetes Care. 18:1446-1451.

70. Diabetes Prevention Program Research Group. 2002. Reduction in the incidence of type 2 diabetes with lifestyle intervention or metformin. N. Engl. J. Med. 346:393-403.

71. Xiang, A.H., et al. 2004. Pharmacological treatment of insulin resistance at two different stages in the evolution of type 2 diabetes: impact on glucose tolerance and $\beta$-cell function. J. Clin. Endocrinol. Metab. 89:2846-2851.

72. Kjos, S.L., and Buchanan, T.A. 1999. Gestational diabetes mellitus. N. Engl. J. Med. 341:1749-1756. 